\title{
Dyckia pontesii (Bromeliaceae, Pitcairnioideae), uma nova espécie do Rio Grande do Sul, Brasil
}

Dyckia pontesii (Bromeliaceae, Pitcairnioideae), a new species from Rio Grande do Sul, Brazil

\author{
Henrique Mallmann Büneker ${ }^{1,2,3}$, Leopoldo Witeck-Neto ${ }^{1} \&$ Kelen Pureza Soares ${ }^{2}$
}

\begin{abstract}
Resumo
É descrita e ilustrada Dyckia pontesii, uma nova espécie de Bromeliaceae (Pitcairnioideae) do Rio Grande do Sul (Brasil). São fornecidos dados sobre seu hábitat, ecologia, distribuição geográfica e afinidades morfológicas, sendo também avaliado o seu status de ameaça segundo os critérios da IUCN.

Palavras-chave: taxonomia, flora austro-brasílica, planta xerófita, Bioma Pampa.
\end{abstract}

\begin{abstract}
Dyckia pontesii, a new species of Bromeliaceae (Pitcairnioideae) to Rio Grande do Sul (Brazil) is described and illustrated. Data on habitat, ecology, geographical distribution, morphological affinities and evaluation about its threat of extinction according to the criteria of the IUCN are provided.
\end{abstract}

Key words: taxonomy, austro-brasilica flora, dryland plant, Pampa biome.

\section{Introdução}

Dyckia Schult. \& Schult.f. é um amplo gênero de Pitcairnioideae (Bromeliaceae), constituído por cerca de 164 espécies (Gouda et al. 2014) encontradas como saxícolas ou terrícolas na América do Sul, especialmente no Brasil Central, Sudeste e Sul, mas também na Bolívia, Paraguai, Argentina e Uruguai (Smith \& Downs 1974). Um recente estudo conduzido por Krapp et al. (2014), apresentou a primeira ampla análise filogenética baseada em caracteres moleculares para Dyckia, confirmando a monofilia do gênero, anteriormente já relatada com base em caracteres morfológicos (Forzza 2001).

No estado do Rio Grande do Sul (Brasil), dentre as Bromeliaceae, o gênero Dyckia é o que possui a maior diversidade específica, sendo citadas 29 espécies (Strehl 2008; Büneker et al. 2013; Forzza et al. 2014). Para este estado sul brasileiro, provenientes de coletas do naturalista Friedrich Sellow, os primeiros táxons relacionados a Dyckia foram descritos por Otto \& Dietrich (1833) e Koch
(1874) (e.g. Dyckia remotiflora Otto \& Dietrich e Prionophyllum selloum K.Koch, esta última Baker (1889) recombinara para Dyckia selloa (K.Koch) Baker). Posteriormente Mez (1919) descrevera D. choristaminea Mez, também proveniente do Rio Grande do Sul e, após a década de 1960, com a publicação de $D$. irmagardiae L.B.Sm. (Smith 1966), novas espécies riograndenses vieram a lume com os trabalhos realizados por Smith (1971, 1988, 1989), Winkler (1982), Irgang \& Sobral (1987), Strehl (1997) e Larocca \& Sobral (2002). Dentre estes trabalhos destaca-se a ampla obra elaborada por Winkler (1982), constituída de chaves e descrições, consistindo no único tratamento taxonômico completo para as espécies de Bromeliaceae do Rio Grande do Sul, nesta foram descritas quatro novas espécies (e.g. D. alba S.Winkl., D. elisabethae S.Winkl., D. retardata S.Winkl. e D. retroflexa S.Winkl.). Trabalhos posteriores (Strehl 2004, 2008) revelaram que o número de novas espécies ainda era grande neste estado, sendo descritas sete (e.g. D. domfelicianenis

\footnotetext{
Colégio Politécnico da Universidade Federal de Santa Maria (UFSM), Av. Roraima, 1000, Camobi, 97105-900, Santa Maria, RS, Brasil.

${ }^{2}$ Universidade Federal de Santa Maria, Herbário do Depto. Ciências Florestais (HDCF), Av. Roraima, 1000, Camobi, 97105-900, Santa Maria, RS, Brasil.

${ }^{3}$ Autor para correspondência: henriquebuneker@mail.ufsm.br
} 
Strehl, D. jonesiana Strehl, D. julianae Strehl, $D$. nigrospinulata Strehl, D. rigida Strehl, D. vicentensis Strehl e $D$. waechteri Strehl). A última nova espécie do gênero descrita para o Rio Grande do Sul, D. strehliana H. Büneker \& R. Pontes (Büneker et al. 2013), assim como a maioria das citadas acima são conhecidas somente para este estado, sendo consideradas endêmicas.

Vê-se que o Rio Grande do Sul abriga uma grande diversidade específica e endemismo de Dyckia, onde novas espécies ainda podem ser localizadas. Uma delas é descrita e ilustrada neste artigo.

\section{Material e Métodos}

Foram coletados espécimes de Dyckia pontesii para estudo em laboratório, cultivo e herborização. Os exemplares vivos foram incluídos na coleção de bromélias do Jardim Botânico do Colégio Politécnico da Universidade Federal de Santa Maria (Rio Grande do Sul). As variações morfológicas da nova espécie foram observadas tanto no hábitat, quanto nos espécimes cultivados. Os dados morfobiométricos quantitativos e qualitativos foram obtidos de indivíduos aleatórios in situ ou ex situ, os florais foram tomados da segunda ou terceira flor, contadas a partir da base da inflorescência. Foi utilizada a terminologia usual para Bromeliaceae, seguindo-se o modelo Smith \& Downs (1977), com as adaptações sugeridas por Scharf \& Gouda (2008), Forzza (2001) e Bernardello et al. (1991). As fotografias foram efetuadas em ambiente natural e a ilustração realizada a partir do material vivo a olho nu, ou com auxílio de estereomicroscópio. Foram consultados os acervos dos herbários HAS, HDCF, ICN, MPUC, PACA, PEL, RB, SMDB, SP, SPF e os acervos digitais dos herbários $\mathrm{B}, \mathrm{G}, \mathrm{GH}, \mathrm{K}, \mathrm{NY}$, P, S, US e WU (acrônimos segundo Thiers 2014). A avaliação do status de conservação da espécie foi feita com base nos critérios da IUCN (2012).

\section{Resultados e Discussão}

Dyckia pontesii $\mathrm{H}$. Büneker, L. Witeck \& K. Soares $s p$. nov. Tipo: BRASIL. RIO GRANDE DO SUL: Pinheiro Machado, saxícola em afloramento rochoso, 12.XI.2013, fl., H.M. Büneker $212 \&$ L. Witeck (Holótipo HDCF; Isótipos RB; SMDB).

Figs. 1a-f, 2a-g

Species morphologice proxima Dyckia elisabethae et Dyckia dusenii. A prima differt marginibus laminaribus cum spinis gracilis, generaliter inconspicuis (vs. spinis latis, conspicuis), vagina candida (vs. spadici), pedunculo et axe inflorescentiae semper viridibus (vs. rubentibus), bracteis floralibus ovato-triangularibus cum apice acuto-attenuato (vs. elipticis cum apice acuminato), floribus sessilibus (vs. pedicellatis), sepalis cum apice acuto, glabris vel sparso-tomentosis solum in apice faciei abaxialis (vs. rotundatis et tomentosis in facie abaxiali) et petalis glabris (vs. petalis villosis). A secunda differt vaginis candidis (vs. spadicibus), bracteis floralibus ovato-triangularibus cum apice acuto-attenuato (vs. ovatis cum apice acuminato), floribus sessilibus (vs. pedicellatis) et filamentis ultra tubum petalo-estaminicum liberis (vs. connatis ultra tubum petalo-estaminicum).

Erva saxícola, rizomatosa; raízes desenvolvidas; roseta foliar $15-55 \mathrm{~cm}$ alt., $18-60$ $\mathrm{cm}$ diam., florida 60-170 cm alt. Folhas ca. 50, as interiores eretas, as exteriores suberetas ou reflexas; bainha suborbicular, branca, brilhante, ca. $3 \times 4,5 \mathrm{~cm}$; lâmina $1,2-3,5 \times 15-35 \mathrm{~cm}$, triangular, rija, suculenta, reta ou arqueada, face adaxial levemente côncava, verde, esparsamente lepidota na base e no restante subglabra ou glabra, face abaxial convexa, longitudinalmente nervado-estriada, albo-lepidota entre as nervuras, ápice com espinho de até $0,6 \mathrm{~cm}$ compr., margens espinhoso-serradas, muitas vezes com espinhos inconspícuos; espinhos patenteaduncos, delgados, mais concentrados na base da lâmina, 0,1-0,4 cm compr., pouco flexíveis, castanhos ou amarelados. Inflorescência axilar, ereta; pedúnculo, robusto, 35-75 × 0,5-1,6 $\mathrm{cm}$, cilíndrico, verde, subglabro a densamente floculoso-tomentoso, tricomas brancos; brácteas pedunculares polísticas, quase igualando ou ultrapassando os entrenós, as basais foliáceas, eretas, ca. $12 \mathrm{~cm}$ compr., margens serradas, face abaxial longitudinalmente nervurado-estriada, ápice agudo, sempre terminado por espinho; brácteas pedunculares da porção superior eretas, adpressas ao pedúnculo, base verde, porção superior castanha, levemente carenadas no ápice, ovadas a largo-elípticas, ápice atenuadoacuminado, espinescente, 1,3-2,6 × 2-6,5 $\mathrm{cm}$, margens inteiras ou com poucos espinhos inconspícuos; porção fértil da inflorescência simples, laxa, 25-75 cm compr., ou composta com 1-7 ramos eretos ou subereto-arqueados locados na base da inflorescência, 25-150 flores; raque verde ou verde acastanhada, subglabra 

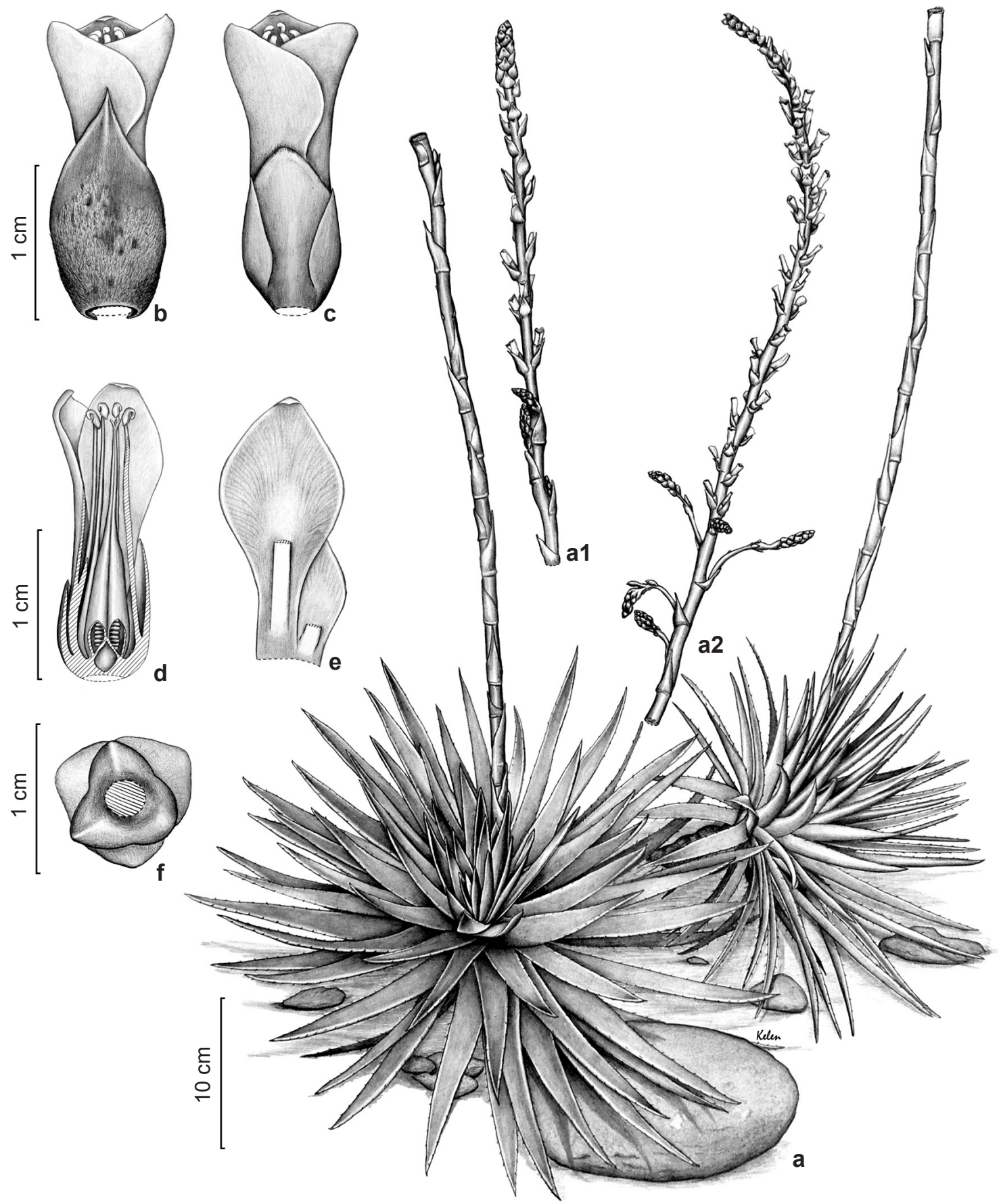

Figura 1 - a-f. Dyckia pontesii H. Büneker, L. Witeck \& K. Soares (H.M. Büneker 212 \& L. Witeck) - a. hábito quando fértil; a1. inflorescência composta em estágio inicial de desenvolvimento; a2. inflorescência composta; b. detalhe da face abaxial da flor com bráctea; c. detalhe da face abaxial da flor; d. corte longitudinal da flor; e. detalhe de uma pétala, sépala e filetes; f. vista inferior da flor.

Figure 1 - a-f. Dyckia pontesii H. Büneker, L. Witeck \& K. Soares (H.M. Büneker 212 \& L. Witeck) - a. habit when fertile; a1. composite inflorescence in early stage of development; a2. composite inflorescence; $b$. detail of abaxial face of flower with bract; c. detail of abaxial face of flower; $d$. longitudinal section of the flower; e. detail of a petal, sepal and fillets; f. bottom view flower. 
a densamente floculoso-tomentosa, tricomas brancos, concentrados principalmente na região abaixo da inserção das brácteas. Brácteas florais oval-triangulares, $1-2,1 \times 1,1-3,5 \mathrm{~cm}$, levemente infladas, levemente carenadas no ápice, base verde, porção superior castanha, subglabras a albo-tomentosas, as da base da inflorescência iguais a superando o comprimento das flores, as do ápice iguais ao comprimento das sépalas, margens inteiras, ápice agudo-atenuado. Flores sésseis, polísticas, ca. $2 \mathrm{~cm}$ compr., na antese suberetas. Cálice levemente zigomorfo; sépalas oval-triangulares, esverdeadas na base, castanhoamareladas na porção superior, $0,6-0,9 \times$ $1,1-1,5 \mathrm{~cm}$, as adaxiais carenadas, a abaxial ecarenada a levemente carenada, adpressas às pétalas, glabras ou esparso tomentoso-lepidotas no ápice da face abaxial, ápice agudo. Pétalas eretas, obtruladas, $1,8-2 \times 1-1,3 \mathrm{~cm}$, amarelas, glabras, ápice arredondado-cuculado. Hipanto ca. $0,2 \mathrm{~cm}$. Estames igualando ou ligeiramente menores que o comprimento das pétalas; filetes branco-amarelados, achatados, retos, com até 0,2 cm larg., conatos $0,2-0,5 \mathrm{~cm}$ acima do hipanto, livres acima do tubo comum com as pétalas, os antessépalos adnatos por $0,1-0,2 \mathrm{~cm}$ às sépalas, os antepétalos adnatos às pétalas por $0,6-0,9 \mathrm{~cm}$. Pistilo 1,8-2,1 cm compr.; ovário ca. $0,7 \times 0,4$ $\mathrm{cm}$, branco-amarelado; óvulos discoides, com ala subfalciforme. Fruto, cápsula, ovóide, castanhos a negros, vernicosos.

Parátipos: BRASIL. RIO GRANDE DO SUL: Pinheiro Machado, saxícola em afloramento rochoso, 22.III.2013, fr., H.M. Büneker 148, R.C. Pontes \& L. Witeck (HDCF); nas proximidades da Vila Torrinhas, saxícola em afloramento rochoso no alto de cerro, 2.V.2014, fr., H.M. Büneker 270, R.C. Pontes, M.D. Ferrarese \& T.S. do Canto-Dorow (HDCF).

$\mathrm{O}$ epíteto específico presta homenagem a Rodrigo Corrêa Pontes, descobridor da espécie, entusiasta e pesquisador das famílias Cactaceae e Bromeliaceae. Dyckia pontesii habita afloramentos e escarpas rochosas conglomeráticas do Bioma Pampa no município de Pinheiro Machado, no sul do estado do Rio Grande do Sul (Brasil), região conhecida como Serra do Sudeste. Os indivíduos de $D$. pontesii compõem touceiras associadas a plantas arbustivas das famílias Apocynaceae (Amblyopetalum coccineum (Griseb.) Malme), Asteraceae (Radlkoferotoma cistifolium (Less.) Kuntze), Malvaceae (Abutilon malachroides A.St-Hil. \& Naudin e A. pauciflorum A.St-
Hil.), Verbenaceae (Aloysia gratissima (Gillies \& Hook.) Tronc.) e herbáceas das famílias Cactaceae (Opuntia sp.), Gesneriaceae (Sinningia macrostachya (Lindl.) Chautems), Iridaceae (Cypella fucata Ravenna), Oxalidaceae (Oxalis spp.) e Poaceae (Aristida sp., Chascolytrum spp., Melica sp., Paspalum spp. e Stipa sp.).

Dyckia pontesii é morfologicamente próxima de Dyckia elisabethae S. Winkl. e Dyckia dusenii L.B.Sm. Difere de D. elisabethae, que ocorre na região leste do Rio Grande do Sul, por apresentar folhas com margem laminar e espinhos delgados, em geral inconspícuos ( $v s$. espinhos largos, conspícuos), bainha branca ( $v s$. castanha), pedúnculo verde ( $v s$. avermelhado), brácteas florais oval-triangulares com ápice agudoatenuado ( $v s$. elípticas com ápice acuminado), flores sésseis ( $v s$. pediceladas), sépalas com ápice agudo, glabras ou esparso-tomentosas no ápice da face abaxial ( $v s$. arredondadas e tomentosas na face abaxial) e pétalas glabras ( $v s$. pétalas vilosas). Difere de $D$. dusenii, que ocorre nos estados do Paraná e Santa Catarina segundo Forzza et al. (2014), e também no Rio Grande do Sul (Büneker, dados não publicados), por apresentar bainha branca ( $v s$. castanha), brácteas florais oval-triangulares com ápice agudoatenuado ( $v s$. ovadas com ápice acuminado), flores sésseis ( $v s$. pediceladas) e filetes livres acima do tubo comum com as pétalas ( $v s$. conatos acima do tubo comum com as pétalas). A espécie também se diferencia das demais por possuir o cálice levemente zigomorfo (Fig. 1f), característica incomum para o gênero, isso porque suas sépalas adaxiais são carenadas e mais próximas entre si do que com a abaxial, que é ecarenada ou levemente carenada.

A nova espécie distribui-se por uma área relativamente ampla (cerca de $2000 \mathrm{Km}^{2}$ ) em comparação a outras espécies do gênero que são restritas a pequenas populações. Além disso é abundante e possui populações preservadas pois na abrangência de sua ocorrência a principal atividade econômica praticada é a pecuária extensiva, ocorrendo em uma grande área rochosa onde é inviável a implantação, em larga escala, de monoculturas. Foram observados níveis significativos de regeneração natural apesar de vegetarem em áreas de pastoreio. Portanto, segundo os critérios da IUCN (2012) a espécie se enquadra na categoria Least Concern (LC). 

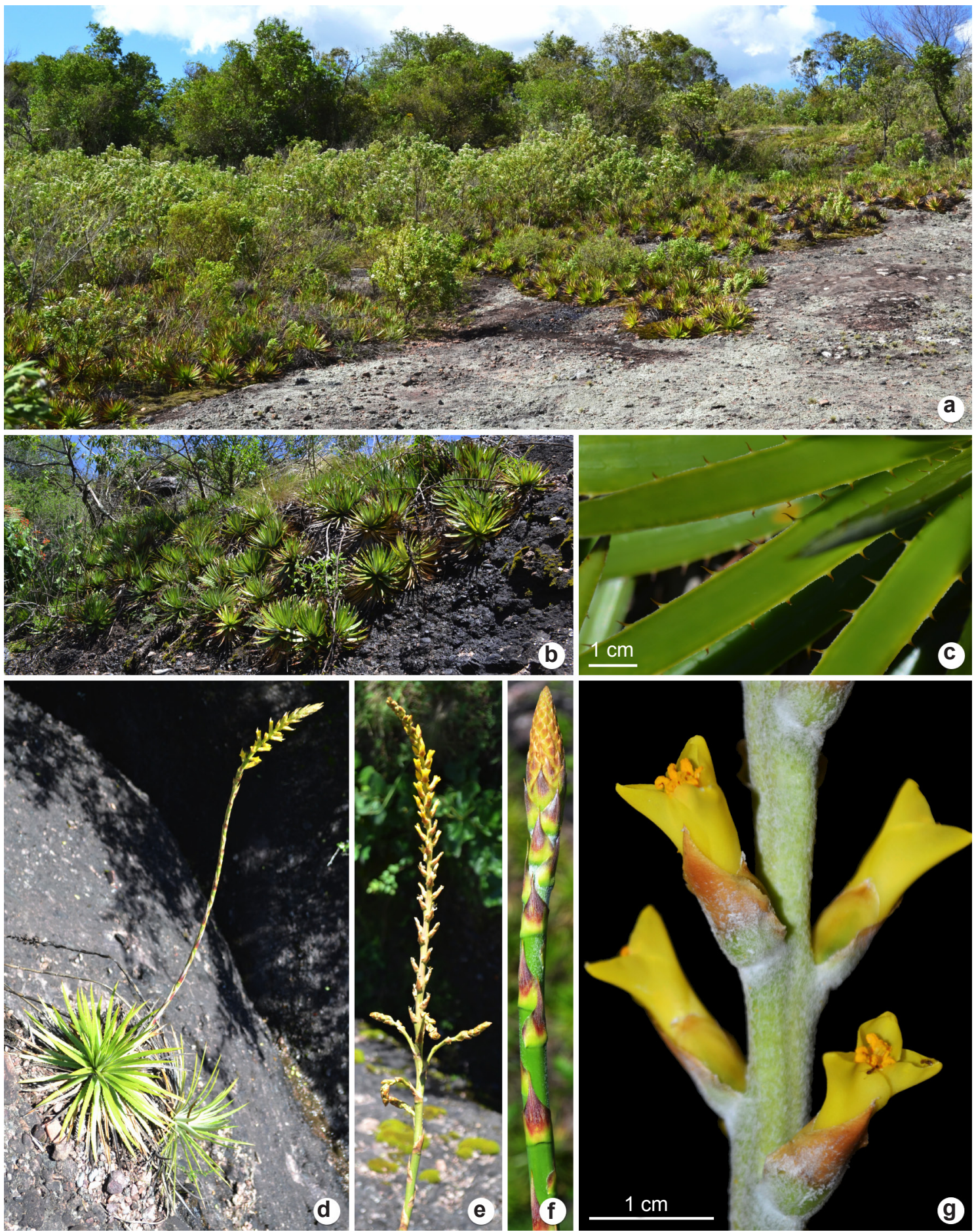

Figura 2 - a-g. Dyckia pontesii H. Büneker, L. Witeck \& K. Soares (H.M. Büneker 212 \& L. Witeck) - a. vista geral da população entremeada por arbustos de Radlkoferotoma cistifolium (Asteraceae); b. aglomerado de plantas em escarpa rochosa; c. detalhe das folhas; $d$. hábito quando fértil; e. detalhe de uma inflorescência composta; f. detalhe de uma inflorescência em desenvolvimento inicial; g. detalhe da inflorescência.

Figure 2 - a-g. Dyckia pontesii H. Büneker, L. Witeck \& K. Soares (H.M. Büneker 212 \& L. Witeck) - a. overview of the population interspersed with shrubs of Radlkoferotoma cistifolium (Asteraceae); b. cluster of plants on rocky escarpment; c. detail of the leaves; d. habit when fertile; e. detail of a compound inflorescence; f. detail of an inflorescence in early development; g. detail of inflorescence. 


\section{Agradecimentos}

Ao Colégio Politécnico da Universidade Federal de Santa Maria e ao coordenador do Curso Técnico em Paisagismo, Marcelo Antônio Rodrigues, que proporcionaram as viagens até os ambientes naturais das espécies do gênero Dyckia encontradas no Rio Grande do Sul, além de também cederem espaço para abrigar a coleção de Bromeliaceae. À professora de latim Leila Maraschin, a contribuição na diagnose. Às professoras do Departamento de Biologia da Universidade Federal de Santa Maria, Thais Scotti do Canto-Dorow e Liliana Essi, as contribuições na organização do artigo, e a Elton Martinez Carvalho Leme, as construtivas discussões taxonômicas.

\section{Referências}

Baker, J.G. 1889. Handbook of the Bromeliaceae. George Bell \& Sons, London. 264p.

Bernardello, L.M.; Galetto, L. \& Juliani, H.R. 1991. Floral nectar, nectary structure and pollinators in some Argentinean Bromeliaceae. Annals of Botany 67: 401-411.

Büneker, H.M.; Pontes, R.C.; Soares, K.P.; Witeck-Neto, L. \& Longhi, S.J. 2013. Uma nova espécie reófita de Dyckia (Bromeliaceae, Pitcairnioideae) para a flora do Rio Grande do Sul, Brasil. Revista Brasileira de Biociências 11: 284-289.

Forzza, R.C. 2001. Filogenia da tribo Puyeae Wittm. e revisão taxonômica do gênero Encholirium Mart. ex Schult. \& Schult.f. (Pitcarnioideae-Bromeliaceae). Tese de Doutorado. Universidade de São Paulo, São Paulo. 208p.

Forzza, R.C.; Costa, A.; Siqueira Filho, J.A.; Martinelli, G.; Monteiro, R.F.; Santos-Silva, F.; Saraiva, D.P.; Paixão-Souza, B.; Louzada, R.B. \& Versieux, L. Bromeliaceae. In: Lista de Espécies da Flora do Brasil. Jardim Botânico do Rio de Janeiro. Disponível em <http://floradobrasil.jbrj.gov.br/ jabot/floradobrasil/FB27674>. Acesso em 17 agosto 2014.

Gouda, E.J.; Butcher, D. \& Gouda, K. 2014 [continuously updated]. Encyclopaedia of Bromeliads Version 3.1. Disponível em $<$ http://botu07.bio. uu.nl/bcg/encyclopedia/brome/>. Acesso em 4 agosto 2014.

Irgang, B.E. \& Sobral, M. 1987. Dyckia agudensis (Bromeliaceae), nova espécie do Rio Grande do Sul, Brasil. Napaea 3: 5-7.
IUCN, Red List Categories end Criteria. 2012. Version 3.1. Switzerland. Disponível em $<$ http:// jr.iucnredlist.org/documents/redlist_cats_crit_ en.pdf $>$. Acesso em 26 janeiro 2014.

Koch, K. 1874. Index seminum in horto botanico berolinensi anno 1873 collectorum. Appendix quarta ad indicem seminum horti botanici Berolinenensis anni 1873, [s.n.], Berlin, pp. 1-7.

Krapp, F.; Pinangé, D.S. de B.; Benko-Iseppon, A.M.; Leme, E.M.C. \& Weising, K. 2014. Phylogeny and evolution of Dyckia (Bromeliaceae) inferred from chloroplast and nuclear sequences. Plant Systematics and Evolution 300: 1591-1614.

Larocca, J. \& Sobral, M. 2002. Dyckia delicata (Bromeliaceae), a new species from Rio Grande do Sul, Brazil. Novon 12: 234-236.

Mez, C. 1919. Additamenta monographica 1919. Repertorium Specierum Novarum 16: 65-79.

Otto, C.F. \& Dietrich, A.G. 1833. Cultur und beschreibung der Dyckia remotiflora, einer pflanze aus der familie der Bromeliaceae. Allgemeine Gartenzeitung 1: 129-131.

Scharf, U. \& Gouda, E.J. 2008. Bringing Bromeliaceae Back to Homeland Botany. Journal of the Bromeliad Society 58: 123-129.

Smith, L.B. \& Downs, R.J. 1974. Bromeliaceae (Pitcairnioideae). Flora Neotropica Monograph 14: 1-662.

Smith, L.B. 1966. Notes on Bromeliaceae, XXIII. Phytologia 13: 84-161.

Smith, L.B. 1971. Notes on Bromeliaceae, XXXII. Phytologia 21: 73-96.

Smith, L.B. 1988. A giant Dyckia mystery. Journal of the Bromeliad Society 38: 248-249.

Smith, L.B. 1989. Another giant Dyckia mystery. Journal of the Bromeliad Society 39: 206-207.

Strehl, T. 1997. Novas bromélias da flora gaúcha I, Dyckia ibicuiensis Strehl sp. nov. Bromélia 4: 14-16.

Strehl, T. 2004. Novas espécies de Bromeliaceae do Rio Grande do Sul, Brasil. Vidalia 2: 19-25.

Strehl, T. 2008. New bromeliads, genus Dyckia, from Rio Grande do Sul, Brazil. Bromeliaceae 42: 8-22.

Thiers, B. 2014 [continuously updated]. Index Herbariorum: a global directory of public herbaria and associated staff. New York Botanical Garden's Virtual Herbarium. Disponível em $<$ http:// sweetgum.nybg.org/ih/>. Acesso em 6 abril 2014.

Winkler, S. 1982. Die Bromeliaceae von Rio Grande do Sul, Südbrasilien. Documenta Naturae 3: 1-90. 